\title{
RESET
}

Recherches en sciences sociales sur Internet

4 | 2015

Les mondes du jeu

\section{La mise en scène de la vie privée en ligne par les adolescents}

Teenagers and their online privacy: How and why they care

\section{Claire Balleys et Sami Coll}

\section{(2) OpenEdition}

Journals

Édition électronique

URL : http://journals.openedition.org/reset/547

DOI : $10.4000 /$ reset.547

ISSN : 2264-6221

Éditeur

Association Recherches en sciences sociales sur Internet

Référence électronique

Claire Balleys et Sami Coll, « La mise en scène de la vie privée en ligne par les adolescents », RESET [En ligne], 4 | 2015, mis en ligne le 21 décembre 2015, consulté le 01 mai 2019. URL : http:// journals.openedition.org/reset/547; DOI : 10.4000/reset.547

Ce document a été généré automatiquement le 1 mai 2019.

(c) Association Recherches en sciences sociales sur Internet 


\title{
La mise en scène de la vie privée en ligne par les adolescents
}

Teenagers and their online privacy: How and why they care

\author{
Claire Balleys et Sami Coll
}

\section{Introduction}

\section{Sphère privée, vie privée et intimité}

1 Que recouvre la notion de vie privée dans les pratiques de sociabilité adolescente médiatisée? Comment les adolescents fabriquent-ils une représentation de leur vie privée sur les réseaux sociaux, et selon quelles logiques? Quelles sont les corrélations existant entre la mise en scène de la vie privée et la distribution du prestige social entre pairs adolescents ? L'objectif de cet article est de répondre à ces questions, en s'appuyant sur différents terrains d'enquête, relevant des médias sociaux, et en mettant l'accent sur la relation qui existe entre les notions de vie privée, d'intimité et d'exclusivité.

2 Pour ce faire, il faut commencer par distinguer la notion de vie privée de la notion de sphère privée. Dans la littérature juridique et parmi les chercheurs qui ont fait de la protection des données leur spécialité, la sphère privée se devine comme une sorte de bulle informationnelle contenant des informations sensibles qu'il faudrait protéger contre les potentielles agressions des institutions privées ou publiques (Stalder, 2002 ; Coll, 2014). La notion de vie privée, en revanche, met davantage en valeur la dimension relationnelle qui relie les individus d'une société ayant droit à une intimité. Contrairement à la sphère privée, la vie privée ne correspond pas à un espace qu'il faudrait délimiter et protéger, mais « existe dans la relation que nous avons avec les autres et non par des signes spatiaux qui la désigneraient » (Jeudy, 2007 : 13).

3 C'est premièrement pour cette qualité dialogique et relationnelle que nous préférons la notion de vie privée à celle de sphère privée, mieux à même de comprendre le rapport adolescent à l'intimité qui est le propos transversal de notre article. Cette précaution, en effet, limite le risque de restreindre la vie privée à une forme de bulle délimitée par une 
frontière à l'intérieur de laquelle des informations seraient ontologiquement privées (Stalder, 2002); ou, en d'autres mots, à une notion singulière d'intériorité qui ne désignerait qu'un rapport de soi à soi (Foessel, 2008). Deuxièmement, nous adoptons une perspective de la vie privée comme s'inscrivant dans un contexte particulier (Nissenbaum, 2009), dans une dynamique sociale avec les membres interagissant dans un même groupe (Coll, 2012), et répondant à l'objectif individuel de se constituer un capital de relations sociales avec ses pairs. Cette perspective est précisément celle qui permet de dépasser l'idée, erronée selon nous, que les adolescents se soucient peu de leur vie privée. Aussi, nous souhaitons défendre une définition de la vie privée qui tienne compte de la valeur des liens intimes et qui offre la possibilité de comprendre le sens des pratiques de négociation, de fabrication et de valorisation de la vie privée en tant que ressource. Sur ce point, nous rejoignons la définition de la vie privée adolescente donnée par Sonia Livingstone (2008), qui met l'accent sur les dynamiques relationnelles entre pairs dans la gestion des informations personnelles. Sur les sites de réseaux sociaux, l'enjeu pour les adolescents est de contrôler la connaissance que les uns ont sur les autres, selon des modalités de négociations qui sont en rapport avec la sociabilité directe. Ceci sachant que les adolescents communiquent prioritairement avec celles et ceux qu'ils connaissent (Cardon, 2009 ; Livingstone, 2008 ; boyd, 2008 ; Metton-Gayon, 2009 ; Balleys, 2014).

\section{Les adolescents et leur vie privée}

La manière dont les adolescents gèrent la représentation et le partage de leur vie privée en ligne fait l'objet d'une littérature scientifique prolixe, qui s'accorde aujourd'hui sur le constat suivant: les adolescents ne s'inscrivent pas dans une logique irréversible de dévoilement de leur intimité, mais davantage dans une gestion consciente et stratégique de leur capital social et symbolique en ligne (Livingstone, 2008; Schwarz, 2010 ; boyd, 2014 ; Litt \& Hargittai, 2014 ; Balleys, 2015a). Aujourd'hui, les mineurs âgés entre 14 et 17 ans administrent plus fréquemment les paramètres de confidentialité d'un site de réseau social comme Facebook que les adultes (Blank, Bolsover \& Dubois, 2014). Plus largement, la prophétie de la fin de la vie privée, annoncée en janvier 2010 par Mark Zuckerberg, semble avoir été définitivement contrecarrée par la littérature sociologique (Coll, Glassey \& Balleys, 2011 ; Tubaro, Casilli \& Sarabi, 2014). Le PDG fondateur de Facebook est même revenu sur sa déclaration et admet aujourd'hui que la croissance de son service dépendra à l'avenir d'une meilleure protection de la vie privée. ${ }^{1}$

5 Selon Antonio Casilli (2013), l'hypothèse de la fin de la vie privée, à laquelle il s'oppose également, a deux origines distinctes. Premièrement, elle a été envisagée pour servir les intérêts économiques de firmes comme Facebook qui utilisent les données privées à des fins commerciales, et ont donc avantage à ce que ces données soient le plus perméables et mobiles possible (voir aussi Glassey \& Coll, 2014). Une seconde origine de cette hypothèse provient, selon lui, d'un «malentendu» conceptuel: «Si, en dépit de ces formes de résistance, l'énonciation de l'hypothèse de la 'fin de la vie privée' a été possible, c'est en raison d'un malentendu foncier relatif aux motivations d'usage des médias sociaux. Trop souvent les analystes et les commentateurs ont pris pour une renonciation intégrale à la privacy ce qui en réalité n'est que l'actualisation de formes de dévoilement stratégique d'informations personnelles à des fins de gestion du capital social en ligne. »(Casilli, 2013 : §14). En d'autres termes, les pratiques de visibilisation et de dévoilement de soi en ligne sont à comprendre comme une façon de faire fructifier le capital social et non pas comme une 
renonciation inconditionnelle à la vie privée. Dans le cas qui nous intéresse ici, l'usage des médias sociaux par les adolescents, les études récentes montrent que les liens amicaux et amoureux tissés entre pairs adolescents constituent les principales ressources mobilisables dans les processus de répartition du prestige au sein des différents réseaux de sociabilité juvénile (Balleys, 2015a).

Deux précautions s'imposent toutefois lorsque l'on parle du capital social des adolescents. En effet, celui dont peut se prévaloir un adolescent auprès de ses pairs ne s'apparente pas tout à fait au capital social tel que défini par Pierre Bourdieu, et ce pour deux raisons. Premièrement, les liens sociaux tissés entre pairs adolescents ne constituent pas « un réseau durable de relations" (Bourdieu, 1980:3), car ces liens, bien que l'objet d'un investissement émotionnel et identitaire fort (Balleys, 2015a), sont dans la plupart des cas non pérennes. Deuxièmement, les capitaux symboliques et sociaux des adolescents, très dépendants l'un de l'autre, sont détachés de leurs capitaux culturels et économiques qui restent encore largement dépendants de ceux de leurs parents. Le capital des adolescents, dans toutes ses dimensions, est un capital en devenir, bourgeonnant principalement entre sa dimension symbolique et sociale. L'affirmation d'Hobbes, « avoir des amis, c'est avoir du pouvoir » (cité par Mercklé, 2004 : 54), résonne particulièrement bien au sein des réseaux de pairs adolescents. Le pouvoir dont il parle, nous préférons ici l'actualiser et le conceptualiser en termes de capitaux social et symbolique. Un capital social peut être accumulé grâce aux "amis ", même s'il reste cantonné à la sphère de sociabilité juvénile et ne permet pas encore de tisser son avenir. Dans le présent, "avoir des amis» (du capital social) permet de gagner du prestige social (ou autrement dit, du capital symbolique) et de gravir les échelons de la hiérarchie sociale adolescente qui a son système de valeurs propre. Il ne permet pas d'obtenir de meilleures notes scolaires, plus profitables pour la construction future de l'âge adulte, mais il revêt une importance essentielle dans la construction d'une vie privée en indépendance de celle des parents et de la famille. D'ailleurs, celui qui obtient les meilleurs résultats scolaires n'est le plus souvent pas celui qui jouit du capital symbolique le plus important auprès de ses pairs (Dubet \& Martuccelli, 1996).

7 Le fait que le capital social adolescent ne soit ni pérenne ni transposable hors de la sociabilité juvénile implique un autre aspect qui le distingue du capital social des adultes : il n'est pas axé sur les liens faibles. En effet, dans les processus de gestion et de valorisation du capital social des adultes, les liens faibles comptent davantage que les liens forts (Granovetter, 1973; Lin, 1995 ; Forsé, 2000). Or, nous allons précisément démontrer le contraire en ce qui concerne les adolescents. Le " sérieux », l'authenticité et l'exclusivitié des liens tissés entre pairs adolescents constituent les principales ressources dans la gestion de leur capital social, essentiellement sollicité pour produire un capital symbolique immédiat. Un capital symbolique dont l'horizon se limitera certes aux relations entre pairs adolescents, qui représentent le groupe de référence et de légitimation de soi pertinent au sortir de l'enfance (Galland, 2001 ; de Singly, 2006 ; boyd, 2008, 2014).

8 Après avoir présenté les deux terrains qualitatifs sur lesquels s'appuie notre argumentation, nous allons commencer par établir la corrélation qui existe entre vie privée, processus d'autonomisation et acquisition du prestige social à l'adolescence. Puis, nous verrons comment la gestion des secrets entre pairs participe à ces enjeux sociaux et identitaires. La complexité des pratiques de mise en scène de la vie privée sur Facebook sera ensuite détaillée, ainsi que les différentes stratégies permettant de visibiliser 
l'intimité tout en la préservant. Finalement, nous montrerons comment certaines pratiques de publication flirtent avec les limites de la transparence, mais sont finalement surtout le résultat d'un travail de mise en scène du lien social, ainsi qu'une manière d'assumer publiquement les rapports d'intimité.

\section{Méthodologie}

\section{Un premier terrain « à triple entrée » méthodologique}

$9 \quad$ Les résultats présentés plus loin sont issus de différents terrains d'enquête qualitative. Une première méthodologie de récolte des données, mise sur pied dans le cadre d'une thèse de doctorat (Balleys, 2011), a été établie sur la base d'un terrain sociologique à triple entrée, c'est-à-dire conjuguant trois méthodes d'investigation d'une même population adolescente. Sur une durée d'une année, du mois de février 2008 au mois de février 2009, les pratiques de sociabilité des élèves de huit classes dans quatre établissements du premier cycle secondaire à Genève, c'est-à-dire de jeunes adolescents âgés entre douze et seize ans, ont été investiguées et suivies. Les établissements ainsi que les filières d'orientation ont été sélectionnés de manière à réaliser un échantillonnage diversifié du point de vue de l'origine sociale, économique et culturelle des adolescents enquêtés. Au cours de cette année ont été réalisés :

- Vingt-quatre entretiens collectifs en situation de réception collective, à savoir quatre focus groupes avec chaque classe. Une première série d'entretiens a été effectuée avec des groupes mixtes, en divisant chaque classe de manière aléatoire, puis une seconde série a été effectuée en divisant les élèves de chaque classe par sexe.

- Des sessions d'observation participante lors d'activités de loisirs organisées par l'école, à savoir l'accompagnement de trois camps scolaires ainsi que de la préparation d'une pièce de théâtre.

- Des sessions d'observation ethnographique sur Internet. Pendant toute la durée d'investigation du terrain, les échanges ayant lieu entre élèves sur leurs blogs respectifs ont fait l'objet d'un suivi régulier ainsi que d'un travail de captation des échanges.

La visée de ce terrain à "triple entrée " était de confronter systématiquement les pratiques de sociabilité directes et médiatisées. Ainsi, ce qui s'échangeait dans le contexte d'un focus groupe pouvait être mis en relation avec des comportements observés en camp scolaire et des propos tenus en situation de face-à-face, corrélés avec les contenus postés en ligne. Ce travail de mise en correspondance des dynamiques sociales et relationnelles en fonction des situations d'interaction était d'autant plus pertinent qu'il avait pour objet un seul panel d'adolescents, dans ce contexte des camarades de classe.

\section{Un second terrain d'observation ethnographique en ligne}

11 Le second terrain d'investigation est une ethnographie en ligne qui s'est déroulée sur une durée de dix-huit mois, entre août 2012 et février 2014. Elle a été réalisée sur les sites de réseaux sociaux Facebook et Ask.fm (Balleys, 2014, 2015b). Les profils dont les contenus ont été investigués présentaient différents modes d'accessibilité. Il s'agissait soit de profils publics, comme ceux du site de réseau social Ask.fm qui sont obligatoirement publics, soit de profils semi-publics, comme dans le cas de profils ouverts aux « amis d'amis » sur Facebook. Pour accéder aux contenus publiés sur ce second type de profil, 
que Dominique Cardon qualifie de « clair-obscurs» (Cardon, 2008), un compte Facebook a été créé afin de pouvoir entrer dans la catégorie des "amis d'amis » prévue par le paramétrage du site. Ce profil n'a pas revendiqué une appartenance sociale ou une identité, et n'a pas non plus sollicité "l'amitié » des usagers. Il était nommé par un pseudonyme ne faisant référence à aucune particularité culturelle et la photo représentait un paysage. Afin de circuler librement sur la multitude des murs Facebook adolescents, ce profil s'est affilié à de nombreux comptes publics, intitulés par exemple : "Les plus belles soirées à Genève"; "Ma meilleure amie, la plus importante ». Ces " amitiés » anonymes ont donné accès à une pléthore de profils Facebook paramétrés de manière à être accessibles aux «amis d'amis ». Dans le respect des règles éthiques de la recherche en ligne (Ess et al., 2002 ; Cora Garcia, Standlee et al., 2009), nous nous sommes tenus à une observation pure (Gold, 2003), sans jamais intervenir ni participer d'aucune manière aux échanges observés. En outre, pour préserver strictement l'anonymat des personnes observées, aucune autre donnée personnelle n'a été récoltée, en dehors de l'âge et du sexe.

Quatre heures de présence hebdomadaire ont été ainsi effectuées pendant une période de dix-huit mois, sur les profils d'adolescents des deux sexes âgés de quatorze à dix-sept ans, selon les indications données sur les profils. L'objectif méthodologique était l'immersion dans l'univers de sociabilité juvénile adolescent, et non, contrairement au premier terrain présenté supra, de suivre les trajectoires d'individus et les liens tissés entre pairs. Par conséquent, les résultats présentés dans cet article, issus du terrain d'observation ethnographique en ligne, prennent sens dans l'articulation et la continuité du terrain « à triple entrée » (Balleys, 2011).

\section{Être «publiquement intime »}

\section{La vie privée adolescente comme enjeu d'autonomisation}

Les résultats montrent que, pour la population adolescente, l'enjeu de l'espace privé est la fabrication d'une vie privée transférable sur un "marché d'échange des intimités " (Sennett, 1979: 19) et non pas la protection d'une sphère privée susceptible d'être envahie par des tiers. Les photos officialisant les nouveaux couples sont légion sur Facebook, et permettent à la fois à ses membres de se rendre visible, et aux pairs de marquer leur approbation. Par exemple, lorsque Valentin, 14 ans, change sa photo de couverture pour une image de lui embrassant sa petite amie, il récolte 100 «j'aime», ce qui représente un record sur sa page Facebook, et reçoit 57 commentaires. Ces chiffres saluent l'avènement de cette relation, et 46 commentaires leur souhaitent «tout le bonheur ", une formule de reconnaissance récurrente dans ce contexte de déclaration du couple sur Facebook. Les relations d'amitié sont également investies d'un important travail de mise en scène et en récit, en particulier entre filles. De longs statuts, puis de longs commentaires en réponse, sont rédigés en honneur à la meilleure amie, faisant référence " aux délires » partagés ensemble, aux sentiments réciproques, à l'exclusivité des liens et aussi souvent aux inimitiés partagées. Par exemple, lorsque Ambre, 15 ans, dédie un long texte à ses deux meilleures amies, l'intimité qu'elle convoque est construite autour de ce qu'elles seules partagent et apprécient ensemble : « tellement de délires que y a seulement nous qui comprenons", mais également autour du rejet d'une "meuf ", contre laquelle elles se sont liguées: «En ce moment, on est TOUTES contre une meuf 
HAHAHAHA !». De fait, l'intimité y est rendue visible dans son existence, mais son contenu est gardé secret, comme ici le type de " délires » auxquels Ambre fait référence, ainsi que l'identité de la personne collectivement détestée. Bien que les adolescents cherchent à préserver un jardin secret face au regard potentiellement intrusif des adultes (boyd, 2014), un jardin intime doit bel et bien être cultivé et se prêter au regard potentiellement valorisant des pairs. Pour comprendre le sens que revêt la notion de vie privée dans les pratiques de sociabilité adolescente médiatisée, il nous faut préalablement comprendre le rôle que jouent les liens forts tissés entre pairs dans le processus de socialisation juvénile, puisque ce sont eux qui se taillent la part du lion dans le capital social d'un adolescent.

Dans la perspective adolescente, être capable de tisser et d'entretenir des liens forts avec ses pairs, c'est-à-dire des liens amicaux et amoureux, est à la fois gage de prestige et de maturité (Balleys, 2015a). En effet, dans l'entre-soi adolescent, la valeur d'un individu, son prestige et son capital symbolique s'évaluent à l'aune du capital social dont il peut se prévaloir. Le capital social ne se mesure pas uniquement en termes quantitatifs, c'est-àdire vis-à-vis de la popularité d'un adolescent, plus ou moins connu et reconnu au sein des différents réseaux de pairs qu'il fréquente. Il se mesure aussi et surtout en termes qualitatifs, c'est-à-dire vis-à-vis de «l'authenticité » et du degré d'intimité que l'individu parvient à faire reconnaître et valider par les différents réseaux de pairs qu'il fréquente. Par exemple, lorsqu'il exprime son capital social en capital symbolique en se vantant dans ses discours, en ligne ou hors ligne, d'avoir «de vrais amis». En somme, les représentations adolescentes de la notion de vie privée peuvent être définies par une certaine forme d'intimité, que nous allons délimiter.

Construire, nourrir et valoriser des liens forts entre pairs adolescents est une activité socialisante fondamentale, au sens qu'elle permet de prendre et d'afficher une distance vis-à-vis de la sphère familiale, et de démontrer par-là qu'on est un "grand " (MettonGayon, 2009). Le lien amical ou amoureux est en effet le lien privilégié à mettre en valeur, contrairement aux liens parentaux et familiaux imposés à la naissance. La capacité de choisir et de séduire les individus qui vont devenir des «très proches ", plus proches que les parents dans tout ce qui touche aux questions intimes, constitue un acte d'autonomisation fort (Balleys, 2015a). L'acquisition de l'autonomie à l'adolescence est ainsi corroborée à l'acquisition d'un capital social propre, constitué de liens forts entretenus avec une sélection de pairs. La notion d'intimité entre pairs adolescents est par conséquent fortement liée à la notion d'exclusivité, puisque ce qui qualifie la relation est le partage d'informations personnelles auxquelles les autres n'ont pas accès. Est intime ce qui est de l'ordre de la confidence, du secret, de ce qui se partage avec celles et ceux que l'on a choisis, à l'exclusion de tous les autres. On comprend que l'intime est, comme le définit Michaël Foessel, « le symbole (...) d'une liberté de choix où les individus expérimentent leur capacité à nouer des relations singulières » (2008: 77).

\section{La valorisation de soi par la valorisation de l'intime}

On trouve une description de cette dynamique de gestion de l'intime il y a plus d'un siècle déjà dans la littérature sociologique. Elle se découvre dans le texte de Georg Simmel, Secrets et Sociétés secrètes (Simmel, 2009 [1906]), qui analyse le pouvoir de valorisation du secret ainsi que la dynamique d'exclusion qui en découle: «D'abord, l'exclusion fortement marquée des autres fait naître un sentiment de propriété non moins fortement 
marqué. Pour bien des natures, ce qui donne son véritable sens à la propriété, ce n'est pas de posséder, au sens positif du terme ; mais elles ont besoin de savoir que les autres sont privés. (...) ce que l'on refuse au grand nombre doit être particulièrement précieux. Et c'est ainsi que la forme du secret donne à la propriété intérieure une valeur caractéristique, parce que dans cette forme, la valeur propre du contenu disparaît assez souvent derrière le fait que les autres n'en savent rien » (Simmel, 2009 [1906] : 42). Si l'on suit cette logique, qui nous permet de reformuler notre propos en d'autres termes, avoir un secret à protéger constitue une ressource symbolique dans la gestion du capital social adolescent, qui attribue de la valeur sociale à l'individu qui le possède, ainsi qu'à ceux qui le partagent. En effet, connaître le secret d'un adolescent, en particulier en matière de sentiments amoureux, implique d'en être suffisamment proche et intime pour qu'il nous donne le droit d'être inclus dans la confidence. Par conséquent, plus le prestige social de celui ou celle qui livre son secret est important, plus l'information est précieuse. Lors des trois camps d'école auxquels nous avons participé, lors de la première enquête, la question de savoir qui était amoureux de qui a pris une place considérable dans les discussions et les préoccupations des élèves. De fait, les spéculations et les tentatives d'extorsion de ladite confidence ont été plus importantes que les idylles elles-mêmes, puisqu'aucun couple ne s'est finalement formé dans aucune des classes. La question n'était considérée comme intéressante que lorsqu'elle impliquait des élèves possédant un fort capital symbolique. En effet, savoir de qui est amoureuse une personne totalement invisible au sein de l'espace relationnel de sa classe (Balleys, 2015a), ne constitue pas un enjeu de prestige. La valeur du secret peut aussi concerner une amitié, en particulier lorsque celle-ci a été déçue. Sur Facebook, de nombreux statuts, plutôt féminins, font état d'une colère ou d'un sentiment d'abandon vis-à-vis d'une amie, ou plus précisément d'une " ancienne meilleure amie », mais sans la nommer. Certaines mentions à l'attention de la personne visée sont faites : «tu te reconnaitras » ou « tu te sentiras concerné ». L'enjeu est alors, pour les camarades qui lisent ce statut, d'être mis dans la confidence. Afin, premièrement, de pouvoir exclure d'en être soi-même la cible et afin, deuxièmement, de maîtriser l'actualité relationnelle et sentimentale du réseau d'amis.

17 Au travers de ces exemples, la dynamique du secret nous semble en effet bien éclairer la problématique à laquelle les adolescents sont confrontés dans les modes de gestion de leur sociabilité, et de manière encore plus complexe depuis l'avènement des médias sociaux, à savoir la valorisation de leur vie privée. Comment rendre visible une vie privée constituée de liens forts entre pairs, afin de pouvoir en retirer du prestige social, tout en préservant ce qui en fait la substance, à savoir le caractère intime, c'est-à-dire exclusif ? Comment faire reconnaître, par les différents réseaux de pairs auxquels l'individu est directement et médiatiquement affilié, la valeur de cette vie privée, c'est-à-dire son caractère légitime et « authentique »? En résumé, si nous suivons Simmel, il faut à la fois en montrer assez pour gagner le prestige lié au secret, mais à la fois pas trop pour que ce secret subsiste. Il s'agit là d'une opération d'équilibrisme complexe qui permet, lorsqu'elle réussit, d'exploiter sa vie privée en construction pour fabriquer du prestige social entre pairs. Dans un mouvement dialectique, ce prestige participe en retour à une meilleure construction de la vie privée.

18 Par le biais des médias sociaux, ce que nous souhaitons appeler la mise en scène de la vie privée en ligne s'effectue au moyen de pratiques d'appropriation, voire de détournement, des paramètres et fonctionnalités prévus par les entreprises comme Facebook ou Ask.fm. Un jeu de fine négociation se met en place entre l'exposition et la dissimulation, dans le 
but de susciter l'intérêt de ses pairs tout en attisant soigneusement leur curiosité, suivant la dynamique du secret relatée plus haut. L'instance de légitimation la plus forte et la plus efficace de ce point de vue est le couple amoureux. Le second terrain rejoint sur ce constat le premier terrain "à triple entrée »: les affaires de couple occupent une place prédominante dans le prestige social et dans l'exercice subtil de la présentation de la vie privée auprès des pairs, car elles sont les meilleurs outils d'affirmation d'une intimité appropriée et autonome. Aussi, les processus d'officialisation du couple en ligne sont particulièrement importants et codifiés, tout comme les références aux tribulations vécues par leurs membres, les «hauts et les bas » qui jalonnent son existence (Balleys, 2015a).

\section{Un double enjeu stratégique}

19 Le site de réseau social Facebook propose deux plateformes de communication : l'une que les adolescents utilisent comme un espace semi-public, le "mur", et l'autre qu'ils utilisent comme un espace privé, l'espace de « discussion instantanée », communément désigné sous l'abréviation «d.i». Ces deux espaces, le «mur» et la «d.i», sont importants à distinguer car les adolescents en font des usages différents et complémentaires. En jonglant d'un mode de conversation public à un mode de conversation privé, selon des procédés déclinables à l'infini, ils parviennent à montrer publiquement qu'ils préservent une vie privée, jouant ainsi avec la tension entre leur intérêt à montrer qu'ils ont une vie privée (des secrets) et l'intérêt à ne pas totalement la dévoiler (Simmel, 2009 [1906]).

Les profils Facebook des adolescents de notre corpus possèdent entre 500 et 5000 « amis ». Il va donc de soi qu'ils ne connaissent pas personnellement toutes les personnes faisant partie de leur réseau Facebook. L'analyse des contenus publiés sur le mur de profils d'adolescents témoigne en effet d'un investissement de cet espace comme d'une scène sur laquelle ils sont en représentation, et qui pourrait s'apparenter à une scène de théâtre, si l'on reprend la terminologie goffmanienne (Balleys, 2014). Le public auquel la mise en scène de la vie privée, soigneusement orchestrée, s'adresse en publiant des contenus est potentiellement extrêmement large, puisque la grande majorité des adolescents ont des profils paramétrés pour être accessibles aux «amis d'amis ». Il s'agit d'une forme d'audience imaginée (Anderson, 2002), en ce sens que les adolescents ne savent jamais qui va lire ou ne pas lire les contenus publiés (boyd, 2008). En revanche, l'accès aux propos tenus dans l'espace de «d.i » est jalousement défendu et réservé à certains élus triés sur le volet. Il s'agit en quelque sorte des coulisses de la sociabilité semi-publique qui se déroule sur le "mur", qui s'organise comme un travail de négociation en équipe (Goffman, 1973), faisant ainsi de la « d.i » un espace strictement privé. Contrairement à la visibilité des contenus publiés sur le «mur», dont la portée n'est pas contrôlable puisqu'ils sont par défaut accessibles à tous les amis Facebook (ainsi qu'à tous leurs amis), les contenus partagés en «d.i» font l'objet d'une opération de sélection des interlocuteurs. Une distinction systématique est réalisée entre les «ayant droit » et les autres, ces derniers pouvant constater explicitement leur propre exclusion du processus de partage d'informations personnelles. Cette opération de sélection des interlocuteurs privilégiés entre dans le jeu de la mise en scène de la vie privée, en augmentant la tension liée au secret et donc au prestige social. L'extrait ci-dessous, issu du profil Facebook d'une adolescente de quinze ans que nous appellerons Sophie, montre comment l'accès à 
l'information sur sa vie privée fait l'objet d'une négociation publique et collective. Elle poste le statut suivant à l'attention de ses 2970 amis Facebook: «J'regrette tout! Il me manque :'/2 ». Vingt-trois personnes cliquent sur le bouton « j'aime » relatif à ce statut et, parallèlement, un échange de commentaires s'active entre Sophie et ses camarades :

Mark : Qui?

Juliette : qui ?

Paul :?

Sophie : Laissez tombez $: \$ 3$

Mark : C $1 \ldots$ ?

Sophie : Naan!!

Mark : A ok

Juliette : Sophie va en D.I

Sophie : Daqk

Ana : Qui ?

Sophie : Vibeer bb !

Stefan :J. C. ? ?

Sophie : Naaaan

Melissa : Soph putain! T'es tellement conne! Pourquoi t'as fais sa putain!

Sophie : Vien en d.i bébé !!

21 Cet échange sert à informer le réseau d'amis Facebook de la qualité des liens qui unissent les uns aux autres, ainsi du degré d'intimité inhérent à ces liens. Les réactions immédiates qui émanent des pairs sont des demandes de complément d'information, en l'occurrence sur l'identité du garçon qui «manque » à Sophie. Alors que cette dernière refuse d'en révéler davantage, "laissez tombez» dit-elle, Mark tente une percée spéculative en proposant des initiales, "C I », ce à quoi Sophie produit un démenti assez vigoureux. Ensuite, Juliette lui demande de passer en « d.i », ce qu'elle accepte aussitôt. On comprend que Juliette est une amie proche de Sophie, puisqu'elle possède l'autorité et la légitimité suffisantes pour lui proposer de transiter vers les coulisses de cet échange, tout comme Ana, invitée à la rejoindre sur le logiciel Viber, où elles pourront poursuivre l'échange sur un mode privé. A contrario, les différents garçons qui interviennent dans l'espace des commentaires, à savoir Mark, Paul et Stefan, sont laissés à leurs vaines tentatives prédictives et sont visiblement maintenus dans l'ignorance. Ils sont autrement dit exposés à la frontière du secret, un fait qui, si on suit Simmel, va donner de la valeur symbolique à Sophie. A la fin du fil des commentaires, l'intervention de Melissa franchit encore une étape dans la démonstration de la proximité qui la lie à Sophie, puisqu'elle la réprimande publiquement de son comportement, faisant allusion à des éléments de 
l'événement que le simple «ami» ou «ami d'ami» Facebook ne peut pas, selon elle, comprendre. La familiarité qu'elle affiche en la traitant de "conne » apporte la preuve d'une forte connivence et provoque un effet de dramatisation sur l'événement en question. Cette complicité partagée se trouve confirmée par la réponse de Sophie : «Vien en d.i bébé ! ».

En postant ce commentaire à l'intention de l'ensemble de son réseau d'amis Facebook, Sophie parvient à rendre visible et à valoriser doublement sa vie privée. Premièrement, elle fait état de regrets et d'un sentiment de manque vis-à-vis d'un garçon, ce qui implique qu'elle a entretenu une relation intime avec un individu du sexe opposé, gage de prestige en soi. En effet, faire état de problèmes de couple, c'est déjà montrer que l'on possède et entretient une vie privée (Balleys, 2015a). Deuxièmement, elle se sert des manifestations de curiosité que son statut a suscité pour marquer son territoire affinitaire, en faisant le tri entre ceux qui auront accès à l'information totale et les autres, et ce de manière visible pour tous. Par ce procédé de sélection de ses amies intimes, Sophie entretient et valorise publiquement sa vie privée, dont les liens d'amitié font partie. Ses amies, comme son précédent petit ami, sont objectivés en ressources monnayables sur le «marché d'échange des intimités » (Sennett, 1979: 19). La réciprocité des échanges est en effet une des dimensions importantes de ce type de marché relationnel et sentimental et Sophie va pouvoir, à son tour, attendre de la part de Juliette, d'Ana et de Melissa des pratiques électives similaires.

\section{Stratégies de contrôle de l'audience}

Comme le montre l'échange de commentaires étudié ci-dessus, il existe différentes options permettant de réduire, et surtout de maîtriser, l'audience de certaines publications : en passant en «d.i », mais également en se donnant rendez-vous sur un logiciel ou une application de partage comme Viber ou WhatsApp. La question qui se pose alors est celle de savoir pourquoi les adolescents jouent un jeu de dupes. En effet, pourquoi n'échangent-ils pas tout de suite et directement par voie de messagerie ce qu'ils réservent de toute façon à l'attention d'un cercle très restreint de proches? Pourquoi se donner des rendez-vous privés depuis des plateformes publiques? A ces questions, nous pouvons maintenant répondre clairement. Afin que la vie privée présente les caractéristiques d'une ressource symbolique, c'est-à-dire qu'elle soit mobilisable dans un processus de valorisation du capital social et d'acquisition d'un statut social élevé vis-àvis des pairs, elle doit non seulement exister, dans les « d.i », par exemple, mais aussi être visible auprès de tous, d'où le recours à toute une mise en scène dans les espaces publics et semi-publics. C'est précisément cette dernière que nous appelons la mise en scène de la vie privée. Le passage en coulisses doit par conséquent s'effectuer face au public des pairs, de manière plus ou moins ostentatoire. Montrer l'intimité la plus exclusive possible au public le plus large possible est par conséquent un moyen d'optimiser la valeur sociale d'un individu, sous les yeux de l'entité investie du pouvoir légitime d'en juger : les pairs. Rendre le couple visible, puisqu'il s'agit de la relation la plus exploitée par les adolescents pour gagner en prestige social (Balleys, 2015a), et surtout légitime aux yeux du public des pairs, c'est avant tout apporter la preuve de l'existence d'une intimité partagée, qui est l'indicateur du « sérieux » vis-à-vis de l'engagement amoureux (Schwarz, 2010).

Un autre moyen utilisé par les adolescents pour apporter la preuve de l'existence de leur vie privée, tout en préservant le contenu intime de la relation de couple, est la capture 
d'écran d'un appareil numérique. Cette pratique consiste à partager l'instantané de ce qui s'affiche à l'écran, que ce soit sous la forme textuelle ou picturale. Elle est utilisée par les adolescents de multiples manières, qui constituent autant de terrains d'expérimentation des frontières entre le public et le privé. Le principe est toujours le même : solliciter le public des pairs comme témoin des sentiments amoureux, des disputes, des émois, des humiliations, bref, des déclarations d'amour et de guerre.

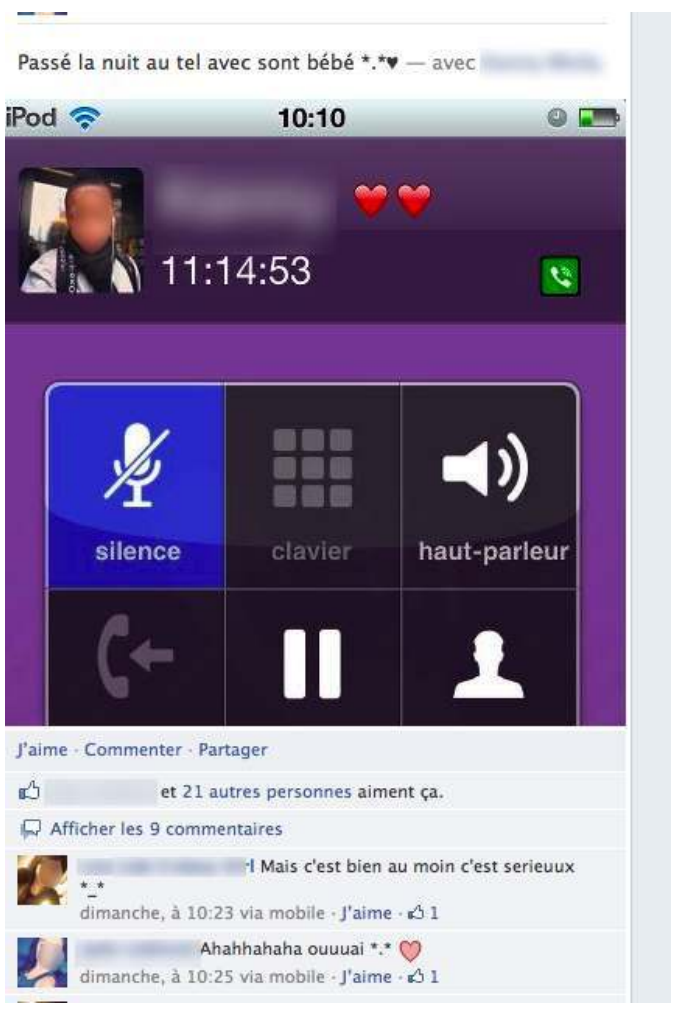

Après avoir passé plus de onze heures au téléphone avec son petit ami, Alizée, 15 ans, procède à la capture d'écran juste avant de raccrocher, alors que le nom de son petit ami ainsi que la durée de la conversation téléphonique s'affichent encore. Ensuite, elle poste cette photo sur son mur Facebook, accompagnée du statut suivant : " passé la nuit au tel avec son bébé », propos qui récoltera vingt-deux clics sur le bouton "j'aime » et neuf commentaires de la part de ses camarades, dont le suivant : «mais c'est bien au moins c'est sérieux ». Les pairs vont ainsi reconnaître et valider la véracité du couple en répondant aux sollicitations d'Alizée. Les captures d'écran permettent successivement l'officialisation d'un couple, puis l'objectivation d'une rupture, opérées par l'acte de publicisation de certains échanges intimes. Elles demeurent cependant toujours le fruit d'un travail de mise en scène du lien social.

Les deux terrains d'investigation montrent que cette mise en scène publique de la vie privée n'est pas limitée aux liens amoureux même si, nous l'avons dit, le couple est au cœur d'enjeux de prestige particulièrement ténus à cette période de la vie, en particulier du fait de sa relative rareté. Certains statuts ludiques sur Facebook permettent de prendre la mesure du faible taux d'adolescents se considérant comme "en couple», en particulier en ce qui concerne les élèves du collège. Par exemple, le statut posté par Maxime, 14 ans - «Aime si t'es célibataire! Commente si t'es en couple, avec le prénom et la date !» - a récolté quatre-vingt-cinq «j'aime », soit autant d'annonces de célibat. Seuls trois adolescents ont posté un commentaire, mentionnant le nom de leur partenaire, avec l'identifiant permettant de rejoindre sa page Facebook, ainsi que la date de leur mise en 
couple. Les liens d'amitié sont beaucoup moins rares et ont moins de valeur symbolique, mais ils sont indispensables à l'acquisition du prestige à l'adolescence. Celui ou celle qui n'a "pas d'ami » est systématiquement mis au ban de la sociabilité juvénile (Balleys 2015a). La logique de divulgation des liens d'amitiés est la même que celle qui prévaut pour l'expérience amoureuse. Il s'agit de montrer qu'une complicité existe, que le lien amical possède aussi, à défaut de la plus grande rareté du lien amoureux, une forme d'exclusivité et d'authenticité. La capture d'écran est là aussi un procédé très utile: il permet en quelque sorte d'apporter la preuve ultime de la véracité de ce qui est partagé. Il s'agit par exemple de publier sur Facebook l'extrait d'une conversation ayant eu lieu sur une application de messagerie, entre deux amies. Dans l'extrait ci-dessous, Diane, 16 ans, décide de rendre public l'échange de messages qu'elle a eu avec Cora, afin de témoigner de la complicité qui les lie :

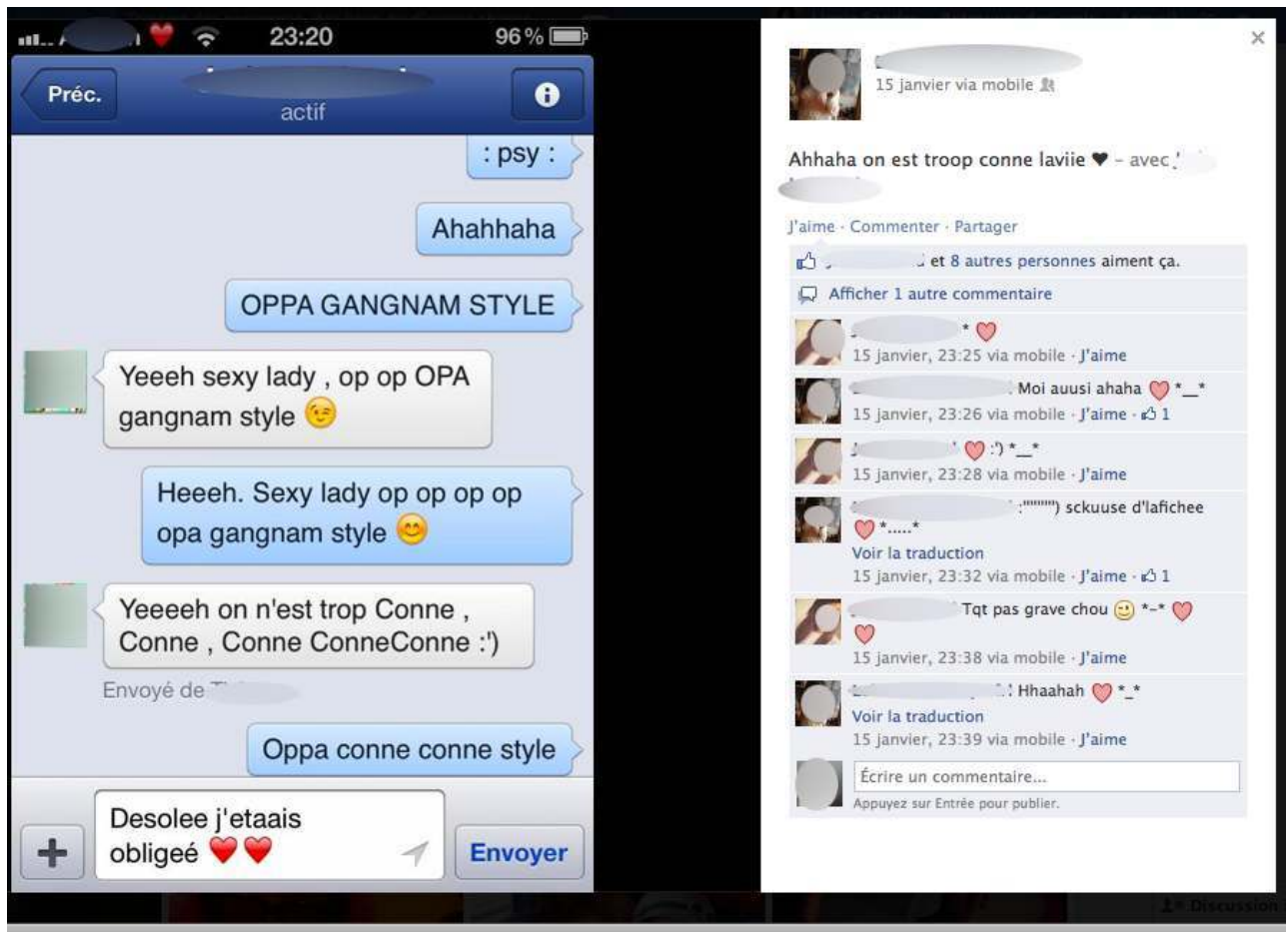

L'extrait montre comment une conversation initialement privée peut se poursuivre sur la plateforme publique que représente Facebook, pour devenir un enjeu de revendication de capital social constitué de liens forts avec les pairs. En effet, le contenu n'a ici rien de « secret » mais constitue pourtant une forme de blague privée, de « délire », comme seuls les bons amis peuvent en avoir, riant ensemble de choses a priori anodines comme ici la parodie d'un tube planétaire. A gauche, on peut voir la capture de la conversation ayant eu lieu sur WhatsApp, où les deux jeunes filles ont repris les paroles d'une chanson populaire pour la tourner en dérision. A droite, on peut voir le statut Facebook que Diane a publié en légende à la capture d'écran, ainsi que les commentaires qui s'en sont suivis. A noter que seules huit personnes ont aimé ce statut, ce qui est peu vis-à-vis d'autres publications de Diane. Par ailleurs, tous les commentaires sont, de fait, le fruit d'un échange entre Diane et Cora. Cette conversation est l'occasion de réaffirmer mutuellement leur complicité, pour Diane en s'excusant auprès de Cora de "l'affiche " qu'elle lui a fait subir, et pour Cora en la rassurant : «t'inquiète pas grave chou ». L'envoi de multiples petits cœurs vient confirmer encore leur attachement. On comprend que l'acte de publication remplit ici une fonction d'officialisation de leur relation d'amitié, 
acte qui vient entériner publiquement l'intimité qui les unit. L'absence de réaction du public des pairs n'indique pas qu'il n'est pas présent. Il existe au moins en tant qu'audience imaginée. Cependant, la publication d'un échange intime permet aussi de valider et de fixer le lien social pour les protagonistes eux-mêmes, ici en l'occurrence Cora et Diane. Cora accepte très bien cette initiative de transfert d'une conversation privée à l'espace semi-public de Facebook, acte qui représente davantage une marque d'affection que la violation d'un espace considéré comme privé.

La capture d'écran et sa publication jouent cependant avec les limites de ce qui est acceptable pour les deux parties impliquées, en particulier pour celui ou celle qui n'a pas choisi de partager un contenu et se trouve devant le fait accompli. Lorsque certains adolescents choisissent de montrer spontanément publiquement l'intérêt et l'affection dont ils sont les objets, sans consulter les personnes concernées, cela peut mener à certaines situations potentiellement conflictuelles. Dans l'extrait de données proposé cidessous, Lola, 15 ans, rend publique la série de messages privés qu'elle a reçu de son ami Sébastien, composée d'une multitude de cœurs, d'émoticônes envoyant des bisous ainsi que de la phrase « Je te pardonne ô ma déesse » :

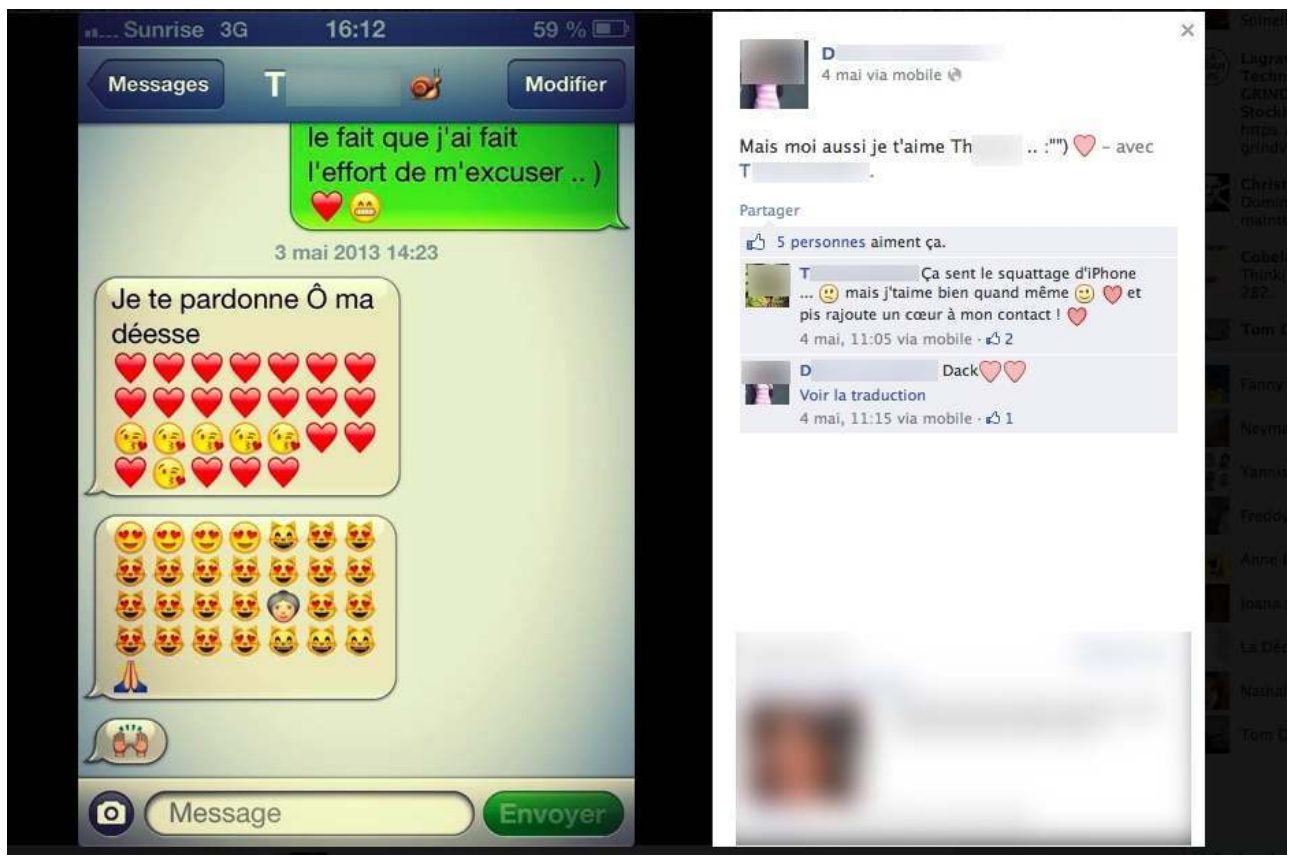

Lola capture cet extrait de conversation et le publie sur Facebook, avec pour légende : « Moi aussi je t'aime Sébastien ». Là encore, la conversation qui suivra sur l'espace des commentaires sera finalement un échange public entre les deux protagonistes de la conversation privée, sans que des tiers n'interviennent. Mais Sébastien relève que cette pratique équivaut à une forme de "squattage ", à savoir la prise de possession de quelque chose qui ne nous appartient pas complètement, et il accompagne cette remarque d'un émoticône avec un visage plutôt mécontent. Cependant, la phrase suivante vient adoucir le propos et rassurer Lola, «mais je t'aime bien quand même », pour enfin lui demander de rajouter un cœur à son contact (plutôt qu'un escargot, en l'occurrence). Il faut noter que Sébastien et Lola ne sont pas en couple au moment de cet échange, mais amis. La publication de cette conversation permet à Lola de communiquer autour de la nouvelle intimité partagée avec Sébastien, qui ne figure pas dans ses précédents statuts Facebook. Par le biais de cet échange, Lola apporte la preuve, au public des pairs comme à Sébastien, qu'elle apprécie cette complicité naissante. En effet, la capture d'écran est la confirmation 
que la relation est assumée socialement, et que l'on n'a pas honte de « s'afficher » comme étant des proches, voire des intimes.

\section{Conclusion}

Le public des pairs joue un rôle d'autorité légitime vis-à-vis du couple dans la dynamique de la mise en scène de la vie privée que nous avons décrite ici. Il a pour fonction de reconnaître et valider l'authenticité des sentiments et de l'engagement investis dans la relation. Cette instance de légitimation exerce une pression relativement forte sur les membres des couples adolescents, les soumettant à une forme de contrôle social dont le champ d'action s'est passablement étendu depuis l'avènement des médias sociaux. Une dialectique extrêmement fine s'observe alors sur les sites de réseaux sociaux, au fil des étapes qui jalonnent la construction de la vie privée des adolescents. Cette dernière est principalement constituée de leur vie amoureuse, quand il y en a une, et de leurs liens d'amitié. Les sites de réseau social comme Facebook constituent des espaces de communication dans lesquels les amis, et à plus forte raison les couples, doivent savoir se rendre visibles, mais également tenir en haleine le public constitué par leurs amis Facebook, d'où les effets de mise en scène et de dramatisation de leur intimité. La gestion de la vie privée entre pairs adolescents à travers les médias sociaux s'apparente ainsi davantage à "une socialité stratégique» (Voirol, 2011: 142) visant à construire et valoriser l'intime en tant que ressource pourvoyeuse de prestige, plutôt qu'à un abandon de la notion de privé, et de la valeur qui lui est associée. Une vision qui fait écho également au texte précurseur de Simmel lorsqu'est décrite la dynamique sociale du secret (Coll, 2012).

Le «marché d'échange des intimités » (Sennett, 1979: 19), élaboré et négocié entre pairs adolescents sur la base du critère d'appréciation de leur vie privée, montre un certain degré de violence symbolique et voit circuler des actes de jugement social. Il présente en effet la particularité d'évaluer la valeur intrinsèque d'un individu sur la base de sa capacité à tisser des liens forts avec ses semblables. Il participe par-là à une stratégie de valorisation du lien social (Foessel, 2008; Voirol, 2011; Illouz, 2006, 2014), puisque le degré "d'authenticité » de soi et de son intimité que l'on parvient à faire valider publiquement devient le critère de reconnaissance de sa valeur propre. Cet article aura permis, en proposant de penser la vie privée comme une ressource mobilisable pour enrichir le capital social et symbolique, et non pas seulement comme une substance à protéger, de montrer dans quelle mesure cette vie privée est traversée par des enjeux de pouvoir et de prise de contrôle. Dans ce contexte, elle peut se comprendre comme un instrument de gouvernance de soi (Foucault, 1984) et un outil d'autonomisation. Mais elle se présente également comme un outil de gouvernance des individus, dès lors qu'une définition normative en est donnée (Coll, 2014). Cette définition, à laquelle les chercheurs participent aussi, à l'instar des juristes et des politiques, entre en tension avec le sens que les acteurs veulent y mettre dans leurs pratiques quotidiennes (Coll, 2015).

La prochaine étape de recherche sur les représentations adolescentes de la notion de vie privée et sur les pratiques de sociabilité qui en découlent, sera de problématiser la contrainte sociale qui s'exerce sur les individus obligés de valoriser publiquement leur vie privée, et par conséquent d'en avoir une. Un futur dispositif de recherche permettra d'observer les adolescents qui sont exclus de ce jeu de valorisation de soi à travers la valorisation des liens forts, à savoir, dans le langage adolescent : les «sans amis ». Une 
première limite des résultats présentés ici est en effet de ne tenir compte que d'une fraction de la population adolescente, la fraction dominante, qui possède une vie privée à mobiliser comme ressource dans la gestion de son capital social. La question des appartenances sociales, économiques et culturelles nécessiterait également une investigation plus individuelle des adolescents enquêtés, ce que ne permet pas le dispositif méthodologique actuel. Par conséquent, un prochain objectif de recherche sera de centrer l'axe méthodologique sur les individus et sur leur expérience sociale du système hiérarchique juvénile, négocié autour du concept de vie privée. Une deuxième limite pourrait être celle de la surinterprétation de la capacité des adolescents à s'approprier les technologies de l'information, un débat sans fin parmi les auteurs et les différents acteurs institutionnels qui semblent plutôt penser les adolescents comme la cible de tous les dangers. Nous avons voulu ici souligner leur autonomie et leur capacité à détourner les usages. Mais dans quelle mesure les adolescents maitrisent-ils indirectement la dynamique sociale du secret, comme nous l'avons suggéré, et disposentils des SRS pour parvenir à leur fin, à savoir de mettre en scène et de valoriser leur vie privée ? Ne sont-ils pas plutôt contraints à se prêter à cette mise en scène ? Voilà une question centrale à laquelle nous aboutissons ici et qui ne manquera pas de guider nos prochaines recherches.

\section{BIBLIOGRAPHIE}

ANDERSON Benedict (2002). L'imaginaire national, Paris, La Découverte et Syros.

BALLEYS Claire (2011). « Comment s'observe la sociabilité adolescente ? Une étude sur trois fronts ", Actes du Colloque international Enfance et Culture, Paris [en ligne], URL : http:// www.enfanceetcultures.culture.gouv.fr/actes/balleys.pdf.

BALLEYS Claire (2014). « De la scène aux coulisses et des coulisses à la scène : les modes d'appropriation juvéniles des réseaux sociaux en ligne », Cultures et Sociétés, 29, pp. 78-84.

BALLEYS Claire (2015a). Grandir entre adolescents. À l'école et sur Internet, Lausanne, PPUR.

BALLEYS Claire (2015b). « Dynamiques d'inclusion et d'exclusion dans la gestion du capital social entre pairs adolescents sur Facebook », Jeunes et médias, les cahiers francophones de l'éducation aux médias, 7.

BLANK Grant, BOLSOVER Gillian \& DUboIs Elizabeth (2014). « A New Privacy Paradox : Young people and privacy on social networks sites ", Paper presented at Annual Meeting of the American Sociological Association, 16 - 19 août 2014, San Francisco, Californie.

BOURDIEU Pierre (1980). « Le capital social », Actes de la recherche en sciences sociales, 31, pp. 2-3.

BOYD dannah (2008). Taken Out of Context. American Teen Sociality in Networked Publics, Berkeley, Université de Californie.

BOYD dannah (2014). It's Complicated. The social life of networked kids, Yale University Press, New Haven. 
CARDON Dominique (2008). « Le design de la visibilité. Un essai de cartographie du web 2.0 », Réseaux, 152 (6), pp. 93-137.

CARDON Dominique (2009). «L'identité comme stratégie relationnelle », Hermès, La Revue, 53 (1), pp. 61-66.

CASILLI Antonio (2013). « Contre l'hypothèse de la « fin de la vie privée », Revue française des sciences de l'information et de la communication, 3.

COLL Sami, GLASSEY Olivier \& BALLEYS Claire (2011). « Building social networks ethics beyond 'privacy' : a sociological perspective », International Review of Information Ethics, 16, pp. 47-53.

Coll Sami (2012). « The social dynamics of secrecy : Rethinking information and privacy through Georg Simmel », International Review of Information Ethics, 17, pp. 15-20.

Coll Sami (2014). « La vie privée comme outil de gouvernance : Surveiller et fidéliser le lien marchand », Les Cahiers du Numérique, 10 (1), pp. 45-68.

Coll Sami (2015). Surveiller et récompenser : Les cartes de fidélité qui nous gouvernent, Terrains des sciences sociales, Zürich, Seismo.

CORA GARCIA Angela, STANDLEE Alecea, BECHKOFF Jennifer \& CUI Yan (2009). « Ethnographic Approaches to the Internet and Computer-Mediated Communication ", Journal of Contemporary Ethnography, 38 (1), pp. 52-84.

DUBET François \& MARTUCCelli Danilo. (1996). À l'école. Sociologie de l'expérience scolaire, Paris, Seuil. ESS Charles et les membres du comité éthique de l'AoIR (2002). Ethical decision-making and Internet research: Recommendations from the aoir ethics working committee, approuvé par AoIR, 27 novembre, 2002 : http://aoir.org/reports/ethics.pdf

foucault Michel (1984). Histoire de la sexualité III : Le souci de soi, Paris, Gallimard.

FOESSEL Michaël (2008). La privation de l'intime : mises en scène politiques des sentiments, Paris, Seuil.

FORSÉ Michel (2000). « Les relations sociales comme ressources », Sciences Humaines, 104, pp. 34-37

GALLAND Olivier (2001). «Adolescence, post-adolescence, jeunesse : retour sur quelques interprétations », Revue française de sociologie, 42 (4), pp. 611-640.

GLASSEY Olivier \& coll Sami (2014). «Le "Gisement de Données” Des Réseaux Sociaux : Le Modèle D’affaire de Facebook. ", in Badillo Patrick-Yves \& Roux Dominique (dir.) Le Futur Est-Il "E-Media ?" , Paris, Economica, pp. 158-67.

GOFFMAN Erwin (1973). La mise en scène de la vie quotidienne. 1. La présentation de soi, Paris, Éditions de Minuit.

GOLD Raymond I. (2003). « Jeux de rôle sur le terrain : observation et participation dans l'enquête sociologique », in Cefaï Daniel (dir.) L'enquête de terrain, Paris, La Découverte, pp. 340-349.

GRANOVETTER Mark S. (1973). « The Strength of Weak Ties », The American Journal of Sociology, 78 (6), pp. 1360-1380.

ILLOUZ Eva (2014). Pourquoi l'amour fait mal. L'expérience amoureuse dans la modernité, Paris, Seuil. ILLOUZ Eva (2006). Les sentiments du capitalisme, Paris, Seuil. JEUDY Henry-Pierre (2007). L’Absence de l'intimité, Paris, Circé.

LIN Nan (1995). « Les ressources sociales : une théorie du capital social », Revue française de sociologie, 36 (4), pp. 685-704. 
LIVINGSTONE Sonia (2008). « Taking risky opportunities in youthful content creation : teenagers' use of social networking sites for intimacy, privacy and self-expression ", New media \& society, 10 (3), pp. 393-411.

LITT Eden \& HARGITTAI Eszter (2014). « Smile, Snap and Share? A Nuanced Approach to Privacy and Online Photosharing ", Poetics (Journal of Empirical Research on Culture, the Media and the Arts), 42(1), pp. 1-21.

MERCKLÉ Pierre (2004). Sociologie des réseaux sociaux, Paris, La Découverte.

METTON-GAYON Céline (2009). Les adolescents, leur téléphone et Internet : «tu viens sur MSN ? ", Paris, L'Harmattan.

Nissenbaum Helen (2009). «Puzzles, Paradoxes, and Privacy in Public » in Nissenbaum Helen, Privacy in Context : Technology, Policy, and the Integrity of Social Life, Standford, Stanford University Press, pp. 103-126.

SCHWARz Ori (2010). « On Friendship, Boobs and the Logic of the Catalogue : Online Self-Portraits as a means for the Exchange of Capital », Convergence, 16 (2), pp. 163-183.

SENNETT Richard (1979). Les tyrannies de l'intimité, Paris, Seuil.

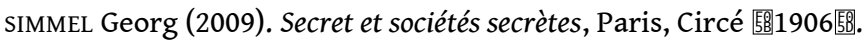

SINGLY François de (2006). Les Adonaissants, Paris, Armand Colin.

STALDER Felix (2002). « Opinion. Privacy is not the antidote to surveillance », Surveillance \& Society, 1 (1), pp. 120-124.

TUBARO Paola, CASILLI Antonio \& SARABI Yasaman (2014). Against the Hypothesis of the End of Privacy, New York/Heidelberg, Springer.

VOIRol Olivier (2011). «L'intersubjectivation technique : de l'usage à l'adresse. Pour une théorie critique de la culture numérique », Denouël Julie \& Granjon Fabien (dir.) Communiquer à l'ère numérique. Regards croisés sur la sociologie des usages, Paris, Presses des Mines, pp. 127-157.

\section{NOTES}

1. Oremus, W. « Facebook Has Totally Changed Its Stance On Privacy. » Business Insider, 26 juillet 2014. http://www.businessinsider.com/facebook-privacy-pivot-2014-7.

2. Emoticône symbolisant un visage qui pleure.

3. Emoticône symbolisant un visage qui fait la grimace.

\section{RÉSUMÉS}

Lorsqu'ils font référence aux usages adolescents des médias sociaux, les adultes considèrent encore très souvent qu'ils n'ont pas de considération pour leur vie privée. Pourtant, une littérature récente montre plutôt que les adolescents maitrisent mieux que les adultes les outils censés protéger leur vie privée. Rebondissant sur ce constat et sur la base de deux enquêtes 
empiriques à entrées multiples, cet article suggère qu'ils ne s'inscrivent pas dans une logique irréversible de dévoilement de leur intimité, mais davantage dans une forme de gestion stratégique. Il montrera comment les liens forts tissés et entretenus entre pairs adolescents sur les sites de réseaux sociaux constituent des ressources dans la gestion de leur capital social, essentiellement sollicité pour produire du capital symbolique.

When referring to the use of social media by teenagers, adults generally still assume that adolescents do not care about their own privacy. However, recent literature demonstrates that adolescents make a best use of privacy protection tools. Starting from this statement, we will argue that they actually take part in some form of strategic management of their social prestige on-line, as opposed to an irreversible process of unveiling their privacy. Drawing on two empirical research studies, the aim of this article is to show how social bonds are established and maintained among peers on social networks, then used as resources by teenagers to improve their social and symbolic capital.

INDEX

Mots-clés : adolescence, vie privée, médias sociaux, intimité, sociabilité

Keywords : adolescence, privacy, social media, intimacy, sociability

\section{AUTEURS}

\section{CLAIRE BALLEYS}

Institut national de recherche scientifique, Observatoire Jeunes et Société, Québec

\section{SAMI COLL}

Université de Lausanne, Institut des sciences sociales 